\title{
Ludwig Holtmeier, Rameaus langer Schatten. Studien zur deutschen Musiktheorie des 18. Jahrhunderts (= Studien zur Geschichte der Musiktheorie, Bd. 13, Veröffentlichungen des Staatlichen Instituts für Musikforschung, Bd. 23), Hildesheim: Olms 2017
}

Schlagworte/Keywords: 18. Jahrhundert; 18th century; basse fondamentale; deutsche Musiktheorie; Georg Andreas Sorge; German music theory; Jean-Philippe Rameau; Johann David Heinichen; Rameaurezeption; reception of Rameau; Satzmodelle; Sorge-Marpurg Kontroverse; Sorge-Marpurg-controversy

Ludwig Holtmeiers Studie Rameaus langer Schatten. Studien zur deutschen Musiktheorie des 18. Jahrhunderts verfolgt drei Kernthesen, die ein gewandeltes Verständnis der Musiktheorie dieses Zeitraums für sich beanspruchen:

1. Rameaus Musiktheorie ist Opfer ihrer Rezeptionsgeschichte: Die einseitige Fokussierung auf den >Rameau des philosophes führt zur Missachtung der praktischen Lehre, die jedoch einen wesentlichen Schlüssel zur spekulativen Theorie darstellt. Erst das Erkennen der Kluft zwischen Werk und Rezeption gewährt den nötigen Weitblick für die musiktheoretischen Alternativen jenseits von Rameau (106-107).

2. Rameaus `langer Schatten $-d$. h. die unilaterale Fixierung auf Rameaus Theorie bzw. auf das, wofür man sie hält - führt zu einem der "größten Versäumnisse der musiktheoretischen Geschichtsschreibung" (109). Sie versperrt den Blick auf die italienische Generalbass- und Partimentotradition. In Johann David Heinichens Kompositionslehre theoretisch erfasst stellt sie einen einzigartigen Gegenentwurf zu Rameau dar.

3. Das Vorgemach der musicalischen Composition des Musiktheoretikers Georg Andreas Sorge ist ein »epochales Werk« (181), das einen immensen Einfluss auf die Nachwelt ausübt. Infolge der Kontroverse mit Friedrich Wilhelm Marpurg und dem slangen Schatten « Rameaus ist diese Schlüsselwerk der ersten Jahrhunderthälfte und mithin ein Teil der italienisch-deutschen Trias-Tradition, der sie angehört, in Vergessenheit geraten.

Diese nicht wenig provokativ formulierten Thesen werden in Holtmeiers Studie - dem beinahe unveränderten Text seiner 2010 an der Technischen Universität Berlin eingereichten Dissertation - in drei verschiedenen Teilen behandelt, die in den folgenden Ausführungen eingehender betrachtet werden sollen.

\section{1. »RECONSTRUCTING THE CLERMONT}

NOTES: GEDANKEN ZUM MUSIKTHEORETISCHEN WERK JEAN-PHILIPPE

RAMEAUS « (11)

Ausgangspunkt für Holtmeiers Beschäftigung mit Rameau sind die sogenannten Clermont Notes, ein Quellenkonvolut, das aus dem Nachlass Rameaus vermutlich in den Besitz Georg Onslows (1784-1852) gelangte und seit den 1960erJahren trotz intensiver Recherche nicht auffindbar ist. Der einzige Zugang zu den Quelleninhalten erfolgte bisher über zwei zusammenfassende Studien des Musikhistorikers René Suaudeau. ${ }^{1}$ Ging dieser von einem Frühwerk Rameaus aus, und folgte ihm die spätere Rameauforschung in dieser Annahme, vertritt Holtmeier die These es sei »schlicht unmöglich, dass [die Clermont Notes] vor der Veröffentlichung des Traité verfasst worden sein könnten« (15).

Diese These - und die Datierung auf die Zeit nach Generation harmonique (1737) - wird 
durch die differenzierte Betrachtung des Entwicklungsstands der in den Clermont Notes enthaltenen Theoreme gestützt, u. a. der Erklärung der Dissonanz durch Verschmelzung zweier Akkorde, der Generierung der diatonischen Ordnung durch den corps sonore und des Theorems der puissance réciproque des vibrations (d. h. der Wirkung einer klingen Seite auf ihre Aliquoten und Aliquanten).

Auf dieser Grundlage erfolgt eine Neueinschätzung der Clermont Notes als eigenständiger Beitrag und als Zugang zu theoretischen Teilaspekten bzw. Gesichtspunkten, die sich in keinem anderen Werk finden. Das betrifft insbesondere die Generierung der Septime durch Substitution der Oktave, die Gleichstellung der Terzverwandschaft gegenüber der Quintverwandschaft, das Prinzip der Substitution und der communauté des notes (d. h. der gemeinsamen Töne). Die Clermont-Notes erscheinen deshalb so wichtig, weil sie, so Holtmeier, die basse fondamentale in der Praxis verankern und das Augenmerk auf die Stimmführungsregeln, die Melodie und den Kontrapunkt lenken.

Holtmeiers Studie kommt das nicht geringe Verdienst zu, sich von dem üblichen Schriftenkanon zu distanzieren oder, besser gesagt, diesen Kanon - durch Aufgabe der Trennung zwischen stheoretischen und spraktischen Schriften - zu erweitern. Das zeigt sich insbesondere in der Konzentration auf den Code de musique (1760), den Guide du compositeur (1759) und die Dissertation (1732). Letzter Traktat wird als »bahnbrechende Schrift, die radikal mit zentralen Annahmen des Traité de I'harmonie gebrochen hat « (4) eingestuft. Die Clermont Notes fungieren bei dieser Neueinschätzung gewissermaßen als Brennglas. Sie bündeln die Strahlen der handwerklichen Interpretationsperspektive, die, so Holtmeier, die gesamte Ramistische Theorie in ein neues Licht rückt. Gewährt diese Kontextualisierung einen nur partiellen Einblick in das theoretische Gesamtœuvre, so gelingt es ihr dennoch Schlüsselkonzepte - wie u. a. ‘double emplois, ssupposition<, 'communauté des notes - in ihrer Entwicklung zu erfassen und in dem gewandelten Licht der Kompositions- und Generalbasspraxis neu zu verstehen. Dies festigt nicht unwesentlich Holtmeiers Grundthese, dass der Schlüssel zum spekulativen Rameau in der praxisnahen Theorie liege, und animiert zu einer umfassenden erneuten Sichtung und Neubewertung von Rameaus Schriften.

Die Fokussierung auf die Clermont Notes kann aufgrund des mit ihr verbundenen Erneuerungspotentials als Stärke der Arbeit betrachtet werden. Gleichwohl ist sie ein fragiler Punkt im sonst robusten Argumentationsgebäude. Holtmeier nimmt damit in Kauf, seine Hypothesen auf die Interpretation zweier Studien zu gründen, die ihrerseits eine Interpretation der Rameau-Manuskripte darstellen. Zweifellos ist es dem Autor gelungen, einen Teil der jüngeren Interpretationsgeschichte kritisch aufzuarbeiten (z. B. die unzweckmäßige Benutzung der Stufentheorie bei Suaudeau). Ebenfalls kann dem Autor nur beigepflichtet werden, dass in Ermangelung der Originalquellen die vorliegenden Studien es dennoch verdienen, als Forschungsgegenstand berücksichtigt zu werden - trotz all ihrer wissenschaftlichen Schwächen, die Holtmeier hervorhebt. Nichtsdestoweniger ist es keineswegs gesichert, dass aus den Informationen, die Suaudeau bereitstellt, Schlüsse gezogen werden »können und dürfen« (29). Ohne Originalquellen oder Transkriptionen sind weder Suaudeaus Informationen noch die darauf (indirekt) fußenden Standpunkte und Ausführungen - z. B. zum Aufbau der Quelle - wissenschaftlich verifizierbar, mögen diese auch noch so plausibel klingen.

Ein weiterer Gesichtspunkt verdient hier der Erwähnung und zwar Holtmeiers Prämisse, dass der Zugang zu Rameaus spekulativer Theorie nur aus einem differenzierten Verständnis der Generalbass- und Kompositionspraxis erfolgen könne. Trotz dieser Kernthese wird die Einbettung der musikalischen Praxis in den übergeordneten wissenschafts- und geistesgeschichtlichen Kontext - und allgemeiner das wechselseitige Verhältnis zwischen diesen beiden Polen - in Holtmeiers Studie nur am Rande thematisiert, z. B. wenn es um die Rameau-Rousseau Kontroverse geht (101-105). Dies soll der ohnehin schon sehr umfangreichen und überaus anregenden Arbeit nicht zum Vorwurf gemacht werden, sondern lediglich dazu dienen, mögliche Perspektiven für weiterführende Studien anzudeuten. 


\section{2. »HEINICHEN, RAMEAU UND DIE}

ITALIENISCHE GENERALBASS-TRADITION: TONALITÄT UND AKKORD IN DER

\section{OKTAVREGEL« (109)}

Der zweite Teil verfolgt das Ziel, den Blick für diejenigen Traditionen und theoretischen Konstruktionen zu schärfen, die durch die Fixierung der Harmonielehre des 19. Jahrhunderts auf Rameau verlorengegangen sind, nämlich die stark an Stimmführung und Kontrapunkt orientierte Generalbass- und Partimentotradition des 17. und 18. Jahrhunderts. Der Oktavregel kommt in dieser Tradition die Rolle eines Katalysators zu. Sie löst den Generalbass aus dem kontrapunktischen Zusammenhang, vereinigt sequenzielle und kadenzielle Momente und spaltet den Generalbass in Wissenschaft und Praxis auf.

In diesem für die Geschichte der Musiktheorie paradigmensetzenden Prozess versteht Holtmeier Johann David Heinichen als Schlüsselfigur. Seine Lehre ist, im Gegensatz zu jener Rameaus, gänzlich aus der Praxis abgeleitet: Die Natur, auf welche sich Heinichen beruft, gründet nicht auf der Proportionslehre oder dem corps sonore, sondern einzig auf den "gebräuchlichen passibus compositionis" (146). Der Oktavregel kommt bei der Identifikation und Einordnung dieser gebräuchlichen Kompositionspattern Systemcharakter zu. Mehr noch: Laut Holtmeier ist sie nicht nur Fortschreitungsschema, sondern auch »Erklärungsmodell « (133), "Mittel der harmonischen Analyse« und "Theorie harmonischer Funktionalität» (115). Was aber genau unter »Funktionalität« verstanden werden soll - offenbar sowohl »dynamische Tendenzen« (141), d. h. Akkordprogressionen, als auch die Bedeutung von Einzelakkorden (127) - und, vor allem, inwiefern die Oktavregel diese Funktionalität tatsächlich theoretisch erfassen und erklären kann, wird im Laufe der folgenden Ausführungen nicht vollkommen klar.

Holtmeier zeigt, dass Heinichens Interpretation der Oktavregel Universalcharakter zukommt, da sie nicht nur Stufenbewegungen, sondern auch Basssprünge erfasst und "funktional zwischen klanglicher Schrittbedeutung und Sprungbedeutung einer Skalenstufe unterscheidet« (133-134). Deutet diese Unterschei- dung fraglos auf eine kontextuelle Interpretation und mithin auf eine variable Funktionalität der Skalenstufen hin, so liefert sie keinen eigentlichen Erklärungsansatz für die auf diese Weise als entweder Schritt oder Sprung schematisierte Differenzierung. Das heißt keinesfalls, dass Heinichens Theorie bar an Erklärungsansätzen wäre. Diese liegen aber eher in den für die Oktavregel auf den ersten Blick sekundären Phänomenen.

Ein wesentliches Kennzeichen der Oktavregel in der Fassung Heinichens, das sich im Übrigen auch in den Bassharmonisierungen Mauritius Vogts $^{2}$ (1719) wiederfindet, liegt in der Benutzung ausschließlich konsonanter Stammakkorde in Grundstellung oder erster Umkehrung. Holtmeier arbeitet hier ein wesentliches Unterscheidungsmerkmal zur Ramistischen Theorie heraus: Während die harmonische Bedeutung der Klänge bei Rameau aus der Differenzierung zwischen Konsonanz und der jeweiligen charakteristischen Dissonanz der dominante und der sou(s)dominante entspringt, entscheiden bei Heinichen die Trias (in Grundstellung) und der Sextakkord über die ,funktionale Ausrichtung` der Klänge«.

Die stufengebundene Zuordnung der (konsonanten) Akkorde ist zweifelsohne signifikant für das Tonalitätsverständnis der Oktavregel: Nur durch die Kenntnis der Tonart - d. h. ihrer Hauptstufe und ihrer diatonischen Skala - wird die Anwendung der Oktavregel möglich. Vor diesem Hintergrund ist es keineswegs ein $\mathrm{Zu}-$ fall, dass gerade bei Heinichen die 'Zirkulation innerhalb der 24 Dur- und Molltonarten zum ersten Mal in einem solchen Maße thematisiert wird. Ebenfalls kann Holtmeiers luzider Deutung nur beigepflichtet werden, dass die Fundierung der Oktavregel auf konsonanten Akkorden einen Aspekt der deutschen triasTradition widerspiegele und sich von Rameaus dissonanzbezogenem Erklärungsschema abgrenze. Nichtsdestoweniger muss berücksichtigt werden, dass die Dissonanz - insbesondere deren "theatralische Resolution ${ }^{3}{ }^{3}$ - eine zentrale Stellung in Heinichens Theoriegebäude einnimmt. Die Oktavregel wird keinesfalls von diesem Kernaspekt ausgespart. So führt Heini-

\footnotetext{
2 Vogt 1719, 118.

Heinichen 1728, 585.
} 
chen nach den schemata modorum, d. h. den Oktavregeln aller Tonarten, an:

\begin{abstract}
Wenn wir nun unsere angegebenen Schemata modorum mit Dissonantien geschickt vermischen wollen, so müssen wir dieses, als ein Haupt-Principium zu voraus setzen: Dass bey natürlich fortgehender Harmonie niemals weder ein ordinairer, noch ein extraordinairer Accord könne angeschlagen werden, davon nicht wenigstens eine eintzige Stimme (wo nicht mehr) in dem folgenden Satze könte liegen bleiben, i.e. legaliter binden, und nachmals resolviren, ohne den Ambitum mo$d i$ in geringsten zu beleidigen. ${ }^{4}$
\end{abstract}

Fungiert die Oktavregel als Kriterium für die korrekte Dissonanzbehandlung, so zeigt sich ebenfalls, dass die Vorbereitung und Auflösung der Akkorde die »natürlich fortgehende Harmonie« indirekt lenken und somit an der Oktavregel selbst teilhaben. Hier entsteht im Keime ein Formalisierungsansatz, der letztendlich doch die Dissonanzbehandlung miteinbezieht.

Ein heikler - und wohl auch bewusst polemisch-kontroverser - Punkt ist Holtmeiers Position zu Rameaus renversement und die mit ihm einhergehende Relativierung der Klangqualitäten: die Aufhebung der "jahrhundertealten und ausdifferenzierten Lehre von den Intervallqualitäten« (125) durch die basse fondamentale entzieht, laut Holtmeier, dem Akkordbegriff »das Moment der Linearität« (126) und bedeutet ein »Verarmen des Begriffs von harmonischer Funktionalität« (127). Dem ist gegenüberzustellen, dass die basse fondamentale in erster Linie keine Umkehrungstheorie, sondern eine Progressionstheorie ist. Informationen über die (potenziellen) kontrapunktischen Linien und Intervallfortschreitungen bleiben mit ihren funktionalen Bedeutungen bis zu einem gewissen Punkt kryptisch in ihren Bewegungen enthalten. An der Stelle des "alten Denkens in Intervallqualitäten « tritt nicht so sehr das »neue Prinzip der Terzschichtung (125), als vielmehr das Ziel, die harmonisch-melodische Dynamik durch eine bedeutungstragende Linie zu erfassen. Nichtsdestoweniger ist Holtmeier darin Recht zu geben - und darauf zielen letztendlich seine Ausführungen ab -, dass die basse fondamentale Rameaus, zumindest in ihrer rezipierten Fassung, wenig bis keine Anhalts- punkte zur konkreten aufführungspraktischen Rekonstruktion des subsumierten Satzes liefert, z. B. im Hinblick auf Stimmlage, -disposition und -dichte.

Vor dem Hintergrund der unangefochtenen Vorrangstellung des Umkehrungsdenkens bis zum heutigen Tag sind Holtmeiers Ausführungen zum Akkord- und Klangbegriff der deutschitalienischen Generalbasstradition ausgesprochen wichtig. Sie machen (erneut) bewusst, dass die Akkordtöne ihre Stimmführungseigenschaften und Hierarchie mit den Umkehrungen verändern können; dass die heute so selbstverständliche Hierarchie zwischen Grundstellung und Umkehrung nicht für alle theoretischen Traditionen gilt, und dass ein sAkkord im Generalbassdenken Heinichens kein einheitliches Gefüge ist, sondern das Resultat von zwei Gerüststimmen, zu welchen sich Füllstimmen gesellen.

Indirekt werfen diese Gesichtspunkte nach Holtmeier die komplexe Frage auf, was einen Klang ausmacht, oder genauer, was einen Klang zu einer Sinneinheit im tonalen Gefüge macht. Allerdings ist die Antwort Rameaus keinesfalls eine so »deutliche und zugleich einfache« wie Holtmeier vorgibt (145). Sie hat jedoch den Vorzug, dynamisch-kontextuelle Elemente (die Art und Weise, wie Akkorde erreicht und verlassen werden) mit statischmorphologischen Aspekten (der konsonante bzw. dissonante Charakter der Akkorde) in einem neuen Erklärungsmodell von hohem heuristischen Wert zu verbinden.

Mit viel Feingefühl gelingt es Holtmeier, wesentliche Aspekte der impliziten Theorie Heinichens - stellvertretend für die sitalienischdeutscher Generalbasstradition - zu rekonstruieren und gelangt so zu dem Ergebnis, dass hinter der basse fondamentale Alternativtheorien verborgen liegen, die die Frage des Klangbegriffs aus anderen Perspektiven (teilweise) beantworten. Vielleicht wäre es angebracht gewesen, gleich in diesem Zusammenhang (und nicht erst in Teil III) Heinichens hochdifferenzierte Resolutionstheorie zu behandeln, liefert sie doch implizite Antworten auf die Frage des Akkordes als Sinneinheit. Auch hätte es sich anbieten können, in diesem deutschitalienischen Kontext auf die Modelle der tabula naturalis im Gefolge von Wolfgang Schoensleder und Johann Andreas Herbst einzugehen. 
Es bleibt jedoch festzuhalten, dass Holtmeiers Ausführungen, zusammen mit anderen Studien der vergangenen Jahre, die Fundamente legen für eine Neuerschließung und Neuwürdigung dieser zum Teil in Vergessenheit geratenen theoretischen Alternativen und Gegenentwürfe.

\section{3. »MARPuRG, SORGE, HEINICHEN UND DIE DEUTSCHE RAMEAU- REZEPTION« (147)}

Im Zentrum des dritten Teils stehen Georg Andreas Sorge und sein Vorgemach der musicalischen Composition, mit welchem, so Holtmeier, »eine andere Art der Musiktheorie beginnt" (182). Sorges Akkordklassifikation, als »herausragendes Ereignis« (184), beruht auf drei Voraussetzungen: einem erweiterten Akkordbegriff, einer rigorosen Ableitung der Akkorde durch ,Verwechslung, und der vollen Autonomie der leitereigenen Klänge. Im Sorge'schen System können mithin auf jeder Stufe des erweiterten diatonischen Raumes Dreiklänge aufgebaut werden, die trotz Umkehrungen die Bedeutung ihres Stammakkords behalten und unabhängig von ihrer Intervallkonstellation als Konsonanzen angesehen werden. Holtmeier betont zu Recht, dass Sorge damit der erste Theoretiker ist, der den Dreiklangsbildungen jenseits der triades perfectae einen solchen Platz einräumt. Ob Sorge ebenfalls als >Begründer der Stufentheorie eingeordnet werden kann, sei hingegen dahingestellt und hängt von den jeweiligen theoretischen Prämissen ab, die für die Stufentheorie als konstitutiv betrachtet werden.

Holtmeier stellt Sorges Dissonanzlehre als herausragende Leistung der Musiktheorie seiner Zeit dar. Im Zentrum dieser Lehre steht die Septime als "Mutter aller Dissonanzen« (218), auf welche jedes Phänomen zurückzuführen ist. Auf dieser Basis entsteht eine hochdifferenzierte Dissonanztypologie, die an oberster Stelle unterscheidet zwischen Septimen, die auf den `Transitus` zurückzuführen sind, und Septimen, die durch Bindungen entstehen. Unter die erste Kategorie fallen u. a. die frei eintretende kleine Septime auf dem Durakkord. Die zweite Kategorie untergliedert sich weiter in gebundene Septimen (= Vorhaltseptimen), die in einen neuen Akkord führen, und retardierende Septimen, die einen Akkord durch Bin- dungen am oberen oder unteren Ende vorhalten. Die Kirnberger'sche Unterscheidung zwischen wesentlicher und unwesentlicher Dissonanz ist somit in der Sorge'schen Theorie vorweggenommen, wie Holtmeier richtigstellt. Zu den wesentlichen Charakteristika des Systems zählt weiterhin, dass die retardierenden Septimen vom Umkehrungsdenken ausgespart sind. Dieses heterogene Element, das auf die Generalbasspraxis zurückgeht, erwächst aus der Konsequenz einer systematischen Rückführung aller Dissonanzen auf die Septime. Ist dem Autor zuzustimmen, dass Sorge zu den Urhebern des "modernen Dissonanzbegriffs zählt» (308), so ist doch nicht außer Acht zu lassen, dass sein System nicht frei von Spannungen ist und verschiedene, zum Teil widersprüchliche Perspektiven nebeneinander bestehen lässt. Sich ein klares Bild dieses Systems zu verschaffen bedarf einiger Zeit und Geduld. Das liegt nicht nur an dessen Komplexität, sondern auch an den Einschüben und Exkursen - z. B. zu Heinichens Lehre der vollstimmigen None -, die Holtmeier in seinen Ausführungen gesondert behandelt.

Dem Sorge'schen System liegt ein neuer Konsonanz- und Dissonanzbegriff zugrunde, der mit »tradierten Vorstellungen radikal bricht« (211). Für Holtmeier besteht dieser Bruch im "fließenden Übergang" der Konsonanzgrade, der an die Stelle einer "groben Trennung" (211) zwischen Konsonanz und Dissonanz tritt. Dem muss man entgegenhalten, dass auch die tradierten Intervallkategorien eine Abstufung der Sonanzgrade implizierten (zwischen perfekter und imperfekter Konsonanz) und dass innerhalb der musica-poeticaTradition die Differenzierung zwischen Dissonanz per se und per accidens auf eine (kontextuelle) Differenzierung der dissonanten Intervalle abzielte. Somit scheint nicht so sehr der fließende Übergang ausschlaggebend für den neuen Klangbegriff zu sein, als ein weiterer, zentraler Gesichtspunkt, den Holtmeier auch thematisiert: Der "Wandel einer HörErfahrung", der einhergeht mit einer "sensualistischen Kritik« der Intervallkategorien (191).

Was sich tatsächlich dahinter verbirgt, ist eine Dissoziation zwischen der empfundenen (dissonanten oder konsonanten) Klangqualität und deren arithmetisch-akustischen Eigenschaften. Erst dieser Bruch führt dazu, dass der 
,Dominantseptakkord als "fast consonant $~^{5}$ (225) betrachtet werden kann. Im selben Atemzug muss allerdings hinzugefügt werden, dass der Bruch zwischen Qualität und Quantität in Sorges Darstellung keinesfalls » radikal « ist.

Sorges Dissonanzbegriff steht, wie Holtmeier zu Recht betont, in der pythagoreischen Zarlinotradition. Diese wird jedoch durch einen gewandelten Bezug zur Empirie um ein neues Naturverständnis erweitert: Die Naturseptime, als ein aus den Tönen des Waldhornes hergeleitetes Phänomen, wird zu einem "Winck der Natur « ${ }^{6}$. Sie bildet den Ausgangspunkt für eine Erklärung mit Anspruch auf Wissenschaftlichkeit und zielt darauf ab, die musikalischen Phänomene auf naturgegebene Hauptgrundsätze zurückzuführen.

Dieser epistemologische Überbau, den Holtmeier nicht sonderlich thematisiert, ist keineswegs nebensächlich für Sorges Position in der Geschichte der Musiktheorie. Das gilt nicht nur für die Oberton- und Untertonspekulationen in der deutschen Musiktheorie des 19. Jahrhunderts, sondern ebenfalls für die Ursprünge der Trias-Tradition, in welcher Johannes Lippius schon auf die Naturtöne der Tubicines als Manifestation der Trias verweist. ${ }^{7}$

Von großer Wichtigkeit für die erwähnte Dissoziation zwischen Qualität und Quantität, die sich bei Sorge herauskristallisiert, ist die Tatsache, dass die Natur zwar als »Wegweiserin«, aber keinesfalls als alleiniges Kriterium der künstlerischen Vollendung betrachtet wird:

Wenn Natur und Kunst einander die Hand bieten, so kommen auch die Sachen, welche die Natur noch etwas unförmlich liefert, in ihre rechte Schönheit. ${ }^{8}$

Das gewandelte Klangverständnis, das sich in der Akkordklassifikation abzeichnet und sich auch in einem neuen "Materialbegriff« (169) äußert, steht somit in enger Verbindung zu ästhetischen, geistesgeschichtlichen und auch wissenschaftsgeschichtlichen Fragestellungen. Holtmeiers Buch kommt das große Verdienst zu, die Originalität, die aus den handwerklichtechnischen Neuerungen der Sorge'schen

\footnotetext{
5 Sorge 1745-47, Bd. III, 359.

6 Ebd., 369

7 Lippius 1610, fol. B4 $r$.

8 Sorge 1745-47, Bd. III, 343.
}

Theorie hervorgeht, erkannt, dargestellt und hervorgehoben zu haben. Um Sorge als »maßgebliche Figur der deutschen Musiktheorie des 18. Jahrhunderts « (9) vollständig zu erfassen, müssten weiterführende Studien - ausgehend von dem von Holtmeier errichteten Fundament - die konzeptuellen Implikationen dieser durch und durch der Praxis verhafteten Theorie systematischer herausarbeiten und in einem breiteren geistesgeschichtlichen Kontext verorten. Es ist zu erwarten, dass diese Perspektive auch dazu beitragen würde, die Spezifizität des intellektuellen und soziokulturellen Kontexts, in welchem Sorges Theorie gedeiht, besser zu erfassen und von jenem seiner Zeitgenossen - u. a. Marpurg und Rameau - abzugrenzen.

Holtmeier zeigt, dass Sorges sekundäre Stellung in der Geschichte der Musiktheorie und seine bislang ausgebliebene Würdigung eng verbunden sind mit seiner »äußerlichen Ähnlichkeit« (178) zu Rameau. Dabei seien Sorge und Rameau im Kern unvereinbar, was Holtmeier, auf relativ engem Raum (hauptsächlich 228-229), anhand der Dissonanztheorie, des Umkehrungsdenken, des Verhältnisses zur Diatonik und des Klangbegriffs veranschaulicht. Ohne im Einzelnen auf die grundsätzlich überzeugenden Argumente einzugehen, seien hier folgende Aspekte betont:

Im Zentrum der Differenz zwischen Rameau und Sorge steht hier wieder der Klangbegriff. Ist der >Normalzustand bei Rameau der dynamische dissonante Akkord, dessen Progressionstendenz eine »Trübung durch Konsonanzen" erfahre, so ist bei Sorge der Ausgangspunkt die statische Trias, die durch Wegnahme und Zunahme von Noten manipuliert und durch Dissonanzen "getrübt« werden könne (229). Holtmeier schließt daraus, dass beide Systeme nicht unterschiedlicher sein könnten. Dieser Gegensatz äußert sich ebenfalls auf der Ebene der Progressionstheorien. Der Ramistischen basse fondamentale - mit ihrer Dissonanzdynamik als Motor - wird eine auf das Grundphänomen der Trias fokussierte Syntax gegenübergestellt:

Harmonische Progression ist auf der elementarsten Ebene nichts als eine Folge von Fundamental-triades, ein endloser vierstimmiger contrapunctus simplex. Dissonanzen können seine Allgegenwart nur verschleiern oder 
sauffhalten`, in letzter Instanz sind sie aber nichts als `Zugaben . (268)

So richtig und überzeugend diese Ausführungen sind, wenn sie auf die Trias harmonica bezogen werden, so fraglich werden sie, wenn man sie auf die gesamte Sorge'sche Theorie überträgt. Es zeigt sich nämlich, dass Sorges Ausführungen im spezifischen Kontext des Durakkords mit der "fast konsonanten « Naturseptime, die Rolle der Dissonanz keinesfalls auf ein aufhaltendes oder verschleierndes Moment begrenzen:

Ob nun wohl diese kleine Septime, die auf der Trompet und dem Wahldhorn das $b^{\prime}$ ist, etwas zu klein oder zu niedrig fällt; so lehret uns doch damit die Natur, daß die erste Dissonanz, so sie darstellet, eine kleine Septime sey und seyn solle, welche, wenn sie auf dem Grunde eines vollkommenen Dreyklangs 4:5:6:2:5:6 oder 1:3:5 ec. ruhet, dem Gehör am aller erträglichsten fällt, und die Harmonie derselben fast mit Gewalt zu einer guten Fortschreitung (Progression) in eine andere ihr nahe verwandte Triadem führet. Denn lasset einmal z. E. $G h d^{\prime} f^{\prime}$ hören, und gebet acht, ob nicht diese Septime gleichsam mit Fingern auf die Triadem c e g, oder auch a $c$ e oder den Sexten-Accord \#G $h$ e weise? so dass man vermittelst derselben durch den ganzen Circkel geführet wird [...]. ${ }^{9}$

Wie Holtmeier zu Recht bemerkt, liegt einer der wichtigsten Unterschiede der Ramistischen und der Sorge'schen Theorien in ihrem Verhältnis zur Diatonik. Unterwerfen sich die Akkorde bei Rameau dem corps sonore und sind sie nur in zweiter Linie an einen diatonischen 'Ort sebunden, so resultieren die Triaden bei Sorge direkt aus der diatonischen Skala und gründen sich auf ihr. So wie der corps sonore und die diatonische Ordnung unvereinbar sind, so sind es laut Holtmeier auch die Theorien Rameaus und Sorges. Hinter dieser wichtigen Erkenntnis verbirgt sich eine weitere, die das Verhältnis zwischen Tonalität und Diatonik betrifft: Ähnlich wie schon die Oktavregel setzt die Stufentheorie (bzw. deren Keime, die sich bei Sorge erkennen lassen) voraus, dass die Tonalität bekannt ist und gewissermaßen präexistiert. Bei Rameau hingegen, induzieren die aus dem corps sonore resultierenden Akkorde

$9 \quad$ Ebd., 341-342. nicht nur die Diatonik, sondern durch ihre Hierarchie und Bezüge auch den tonalen Raum.

Alles in allem kann Holtmeiers These nur zugestimmt werden, dass Rameaus und Sorges Systeme sich in ihren Grundsätzen stark voneinander abgrenzen. Einmal abgesehen von dem 'kreativen Input‘, den Sorge durch die indirekte Rezeption Rameaus über Mattheson erhalten hat, zeugt das Vorgemach von einer in ihrer Originalität bemerkenswerten Neuinterpretation der italienisch-deutschen Traditionen. Nach einer oft mühsamen und hochdifferenzierten Erörterung der individuellen Theoreme hätte die Gegenüberstellung beider Theoretiker sicherlich plastischer und auch exponierter im Werk ausfallen können, was jedoch nur wenig an deren inhaltlicher Pertinenz ändert.

Es sei abschließend auf den letzten Teilaspekt der von Holtmeier vorgebrachten Thesen eingegangen: die Marpurg-Sorge-Kontroverse und ihre Konsequenzen für die Rezeption der Sorge'schen Theorie. Holtmeier lenkt das Augenmerk auf die historische Bedeutung dieser Auseinandersetzung - wohl eine der wichtigsten und folgenreichsten der Musiktheorie des 18. Jahrhunderts -, die letztendlich zu Sorges Verschwinden aus der Musiktheorie führte. Anhand eines close readings der Schlüsselstellen der Marpurg'schen Attacken gegen Sorge mutmaßt Holtmeier, dass die Kontroverse auf einen Plagiatsvorwurf Sorges gegen Marpurg zurückgehe. Sorges (berechtigte) Bezichtigung, die Akkordklassifikation und Resolutionslehre aus dem Vorgemach plagiiert zu haben, versucht Marpurg von sich zu wenden, indem er Sorge seinerseits beschuldigt Rameau zu plagiieren. Marpurgs Strategie, in welche sich auch die Übersetzung Rameaus in der Fassung d'Alemberts fügt, läuft somit darauf hinaus, seine eigene Theorie unter die Autorität Rameaus zu stellen, um ihre Abhängigkeit von Sorge zu verbergen. Dabei sei die Marpurg'sche Theorie im Kern »nicht nur anti-ramistisch, sondern durch und durch sorgisch« (317).

Dass Sorges Name als Theoretiker durch den Ausgang der Kontroverse wenn nicht vernichtet, so doch beträchtlich geschmälert wurde, ist historisches Faktum. Ebenfalls ist es vollkommen denkbar (wenn auch nicht gesichert), dass der Ausgangspunkt dieser Feindschaft in dem von Holtmeier identifizierten Plagiatsvor- 
wurf liegt. Die 'Strategie Marpurgs, sich hinter den Schutzschild Rameaus zu stellen, um Sorges Anschuldigungen abzuwehren, ist in Holtmeiers Darlegung zwar weitgehend plausibel. Jedoch reichen die nur knappen Ausführungen zu Marpurgs Theorien in Holtmeiers Werk nicht aus, um diese Hypothese im Detail zu überprüfen. Erst eine systematische und chronologisch differenzierte Neuerschließung aller in diesem Zusammenhang stehenden Schriften könnte dazu beitragen, die nötigen Anhaltspunkte zu erfassen, die die vermeintliche Strategie Marpurgs endgültig belegen.

\section{4. »AUSKLANG UND AUSBLICK« (319)}

Ob Sorge tatsächlich Rameau und Heinichen »übertrifft« (181), möge jeder Leser für sich entscheiden und ist sicherlich auch nicht die richtige Ausgangsfrage, um den in Rameaus Schatten stehenden Traditionen gerecht zu werden. Wie auch immer, Holtmeiers Werk trägt sehr viel dazu bei, diese Traditionen freizulegen, ihre Bedeutung fasslich zu machen und einen wichtigen und in seinen Anschauungen hochoriginellen Musiktheoretiker zu rehabilitieren. Dabei stellt Holtmeier nicht geringere Ansprüche an seine Leser*innen als Sorge und Rameau selbst. Der virtuose Umgang mit theoretischen Konstruktionen, die stichhaltige und zuweilen schneidend-provokative Argumentation sowie Holtmeiers prägnante Formulierungen tragen jedoch viel zu einer anregenden und bisweilen begeisternden Lektüre bei.

Der Bogen zu Rameaus praktisch-handwerklicher Lehre schließt sich am Ende nur implizit und auch bleiben einige Punkte der deutschen Rameaurezeption im Dunkeln. Das ändert jedoch nichts an der Bedeutung dieses in sich abgerundeten Werkes, sondern animiert vielmehr dazu, auf diesem stabilen Fundament weiterführende Studien zu gründen, u. a. zum Einfluss von Marpurgs - und damit gegebenenfalls auch Sorges - Akkordlehre auf die französische Theorie, oder zum Einfluss der italienischen Musiktheorie auf die deutsche in der ersten Hälfte des 18. Jahrhunderts. Holtmeier legt mit seinem Werk den Grundstein zu einer neuen, auf einem grundlegenden Quellenstudium basierenden europäischen Geschichte der Musiktheorie.

\section{Christophe Guillotel-Nothmann}

\section{Literatur}

Heinichen, Johann David (1728), Der GeneralBass in der Composition [...], Dresden: Selbstverlag.

Lippius, Johannes (1610), Disputatio musica [...], Wittenberg: Gormanus.

Sorge, Georg Andreas (1745-47), Vorgemach der musicalischen Composition, Lobenstein: Selbstverlag.

Suaudeau, René (1958), Le premier système harmonique (dit Clermontois) de JeanPhilippe Rameau, Clermont-Ferrand: École Nationale de Musique.
Suaudeau, René (1960), Introduction à l'harmonie de Rameau. Clermont-Ferrand: École Nationale de Musique.

Vogt, Mauritius (1719), Conclave Thesauri Magnaee Artis Musicae [...], Prag: Labaun. 
Guillotel-Nothmann, Christophe (2019): Ludwig Holtmeier, Rameaus langer Schatten. Studien zur deutschen Musiktheorie des 18. Jahrhunderts (= Studien zur Geschichte der Musiktheorie, Bd. 13, Veröffentlichungen des Staatlichen Instituts für Musikforschung, Bd. 23), Hildesheim. Olms 2017. ZGMTH 16/1, 143-151. https://doi.org/10.31751/1010

(C) 2019 Christophe Guillotel-Nothmann (Christophe.Guillotel-Nothmann@cnrs.fr) Centre National de la Recherche Scientifique - Institut de Recherche en musicologie

Dieser Text erscheint im Open Access und ist lizenziert unter einer Creative Commons Namensnennung 4.0 International Lizenz.

This is an open access article licensed under a

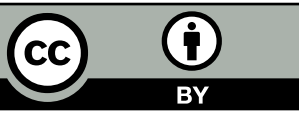

Creative Commons Attribution 4.0 International License.

eingereicht / submitted: 15/05/2019

angenommen / accepted: 01/06/2019

veröffentlicht / first published: 30/06/2019

zuletzt geändert / last updated: 30/06/2019 